\title{
Macromodelling for Analog Design and Robustness Boosting in Bio-Inspired Computing Models
}

\author{
J.Cuadri*, G.Liñán, E.Roca, A.Rodríguez-Vázquez \\ Instituto de Microelectrónica de Sevilla, Centro Nacional de Microelectrónica, Avda. Reina \\ Mercedes s/n, Campus Universidad de Sevilla, E-41012 Sevilla (Spain)
}

\begin{abstract}
Setting specifications for the electronic implementation of biological neural-network-like vision systems on-chip is not straightforward, neither it is to simulate the resulting circuit. The structure of these systems leads to a netlist of more than 100.000 nodes for a small array of $100 x 150$ pixels. Moreover, introducing an optical input in the low level simulation is nowadays not feasible with standard electrical simulation environments. Given that, to accomplish the task of integrating those systems in silicon to build compact, low power consuming, and reliable systems, a previous step in the standard analog electronic design flux should be introduced. Here a methodology to make the translation from the biological model to circuit-level specifications for electronic design is proposed. The purpose is to include non ideal effects as mismatching, noise, leakages, supply degradation, feedthrough, and temperature of operation in a high level description of the implementation, in order to accomplish behavioural simulations that require less computational effort and resources. A particular case study is presented, the analog electronic implementation of the locust's Lobula Giant Movement Detector (LGMD), a neural structure that fires a collision alarm based on visual information. The final goal is a collision threat detection vision system on-chip for automotive applications.
\end{abstract}

Keywords: bioinspired models, vision system on-chip, analog design, collision avoidance

\section{1-INTRODUCTION}

The electronic implementation of bio-inspired computing techniques is gaining attention as a hot topic from many different fields of research. The reason is clear: through thousands of years of evolution nature has produced extremely efficient systems whose computing capabilities for solving real-life problems are far beyond those of the most advanced computers. In the field of artificial vision, interest in models inspired in nature also is arising strongly nowadays. Traditional processing techniques and architectures have shown to be suitable for some applications, but their needs in processing speed and power consumption make their integration into restrictive environments very difficult. Thus, a change of mind in the design of electronic solutions for these problems is mandatory. Analysing natural systems, and trying to implement them or extract a methodology from their structure is a promising path for science.

Analog electronics reveals to be an adequate choice to implement these systems, because it leads to an optimum use of silicon area, low power consumption and high processing speed. The first step in traditional analog electronic design is to establish a set of specifications the implementation must meet. Non idealities in the electronic implementation introduce undesired effects that may affect drastically the ideal behaviour of the circuit, and therefore the initial set of specifications. Additionally, due to the complex structure of biological models, it is not straightforward to set this specifications up in an analytical way. Furthermore, biological models usually include a number of parameters that can be tuned, and whose influence on the performance is not always analytically described. Tuning them and/or making modifications on the structure of the model for it to work properly would also be desirable, taking into account electronic influences that will lead to a much more accurate choice of their value. Thus, simulation is mandatory, including the non ideal effects on which setting up the specifications. The architectural characteristics of this kind of systems, normally representing a complex neural network scheme, make their low level electrical simulation very difficult and time consuming, with traditional electronic design environments. Even for small inputs (100x150 pixels) the number of nodes to be computed in an electric

* Mail: cuadri@imse.cnm.es, Phone: +34-955-056666, Fax: +34-955-056686 
simulation may rise beyond 100.000. This would imply the need of a huge amount of computational resources. Therefore, a high level description of the electronic implementation (i.e., a macromodel) is needed to make feasible the behavioural simulation of these systems, prior to the beginning of the circuit design stage.

In this paper a methodology to successfully make the translation from the mathematical description of the biological model to the specifications for the hardware implementation is proposed. An elemental block decomposition of the cells that compose the system is made, and non ideal effects as mismatching, noise, leakages, supply degradation, feedthrough, and the influence of temperature of operation are included in a macromodel. A new previous step in the flux diagram of the analog electronic design is included, to face this particular problem. As a case study, the analog integration in silicon of a collision avoidance system for automotive applications is studied, based on the previously modelled ${ }^{1}$ locust visual system. Locusts have a neuronal structure called Lobula Giant Movement Detector (LGMD) that selectively responds to looming objects that are supposed to be approaching on a collision course. The input to the system is the visual information (images), and the output is a collision alarm. When it fires, an evasive manoeuvre and/or trajectory correction is accomplished. This allows those animals to fly in huge and very dense swarms without colliding with each other, apart from escaping from hazardous situation as approaching predators. Even though in this case initial mathematical description is relatively simple, it still presents some problems for its electronic implementation, as it will be explained later.

The paper is organized as follows: in section 2 the methodology followed to develop the analog electronic macromodel is described. In section 3 the locust's LGMD is fed into the design flux as a case study. Finally in section 4 conclusions will be drawn out.

\section{2-MACROMODELLING OF BIOLOGICALLY INSPIRED ANALOG CIRCUITS}

The proposed methodology to make the translation from biological models to circuit-level specifications is depicted in Fig. 1. From the description obtained by biologists, normally consisting of complex systems of differential equations, a computational model must be extracted. This must be an adaptation of the model to the computing resources available in standard analog electronics, with the main objective of using a minimum set of operators. These operators should afterwards be individually identified, and their implementation defined, according to area, power consumption and accuracy criteria. In the field of vision, the general structure of an analog processing chip consists of an array of cells, each one of them including sensory circuitry, accompanied by processing units that will perform the requested operations with the incoming data and, when necessary, memories. Each of these elements will constitute a building block of the macromodel. A possibility for the composition of a cell at block level can be seen in Fig. 2a). The processing is implemented by multiplexing in time the use of the analog operators, through the common bus inside each cell. After the definition of the building blocks, the non idealities they present can be described, taking into account the experience of circuit designers, whose advice is also mandatory in the step of identification and implementation of operators. Once the macromodelling of the circuit at block level is done, the system level simulation can be accomplished to characterize the way in which electronics non idealities will alter the expected behaviour of the model. When necessary, redesign of the analog operators or redefinition of the macromodel can be done to improve the results obtained by simulation, aiming to the robustness of the final analog implementation, but without a traditional electrical simulation.

Intrinsic to the analog implementation of the operators and memories, there are a number of non idealities that alter the final behaviour of the system. First of all, the photosensors should be analysed. The simplest image acquisition mechanism consists of a photodiode and its parasitic capacitor, controlled by a reset signal through a switch (Fig. 2b)). When the reset signal activates, the parasitic capacitor of the photodiode is charged to a fixed reset voltage Vdd. Then, when reset disactivates, the sensing process begins, current flows through the photodiode proportionally to the intensity of incident light on it, and causes progressive discharge of the pre-charged capacitor. When the exposition time ends, the voltage of the parasitic capacitor is read by an output buffer, and stored as the actual pixel value ${ }^{6}$. There are two main sources of errors in the photosensor layer: the voltage supply curvature and the saturation of the photoreceptors. The voltage supply degradation consists of a spatial variation of Vdd at each pixel, due to the finite resistance of the wires used to distribute this supply. One of the possibilities to model that is to create a resistive grid like the one in Fig. 3a) and simulate it (Fig. 3b)). 


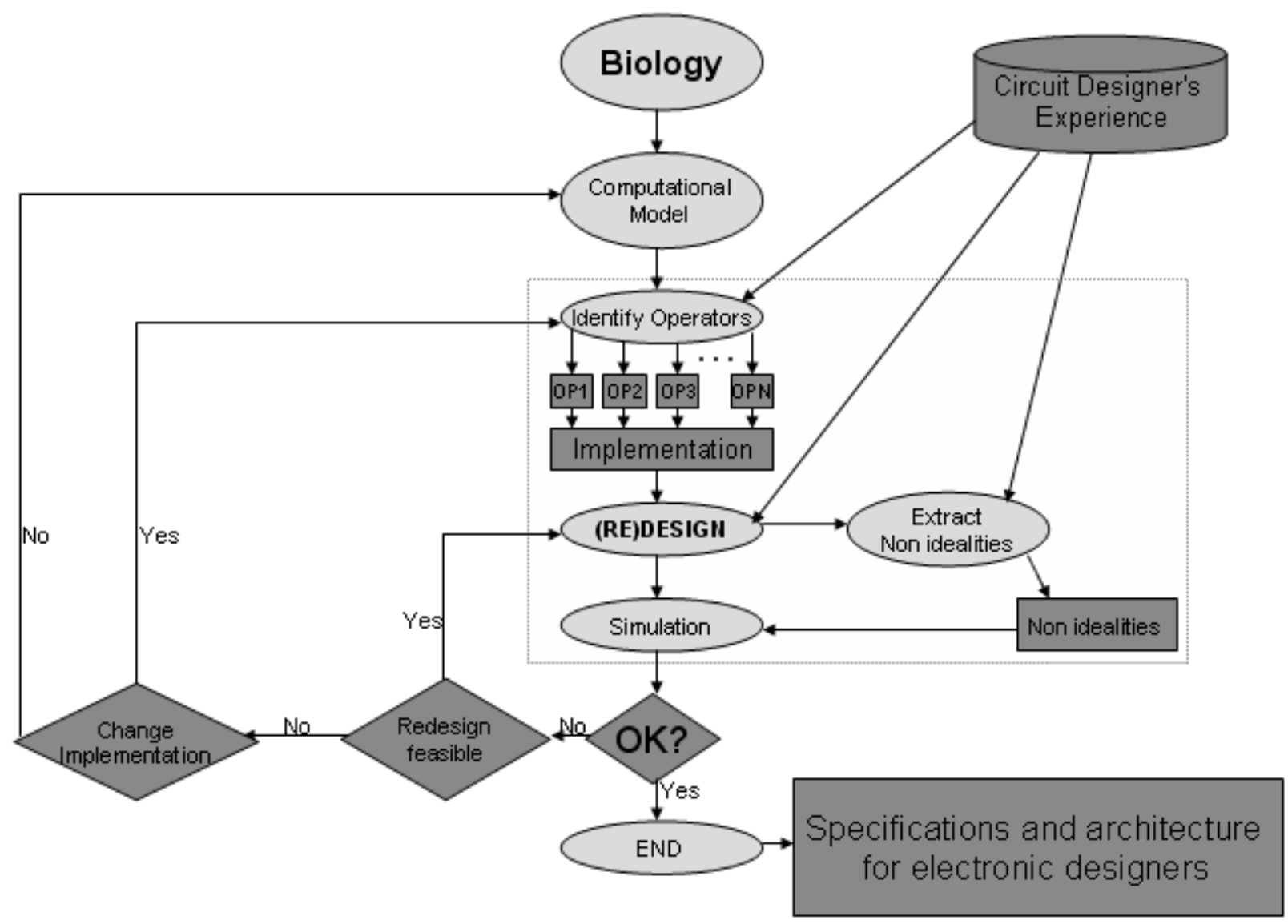

Figure 1: Flux diagram of the design process for the case of biological modelling. In the box, the stages in which this paper incides.

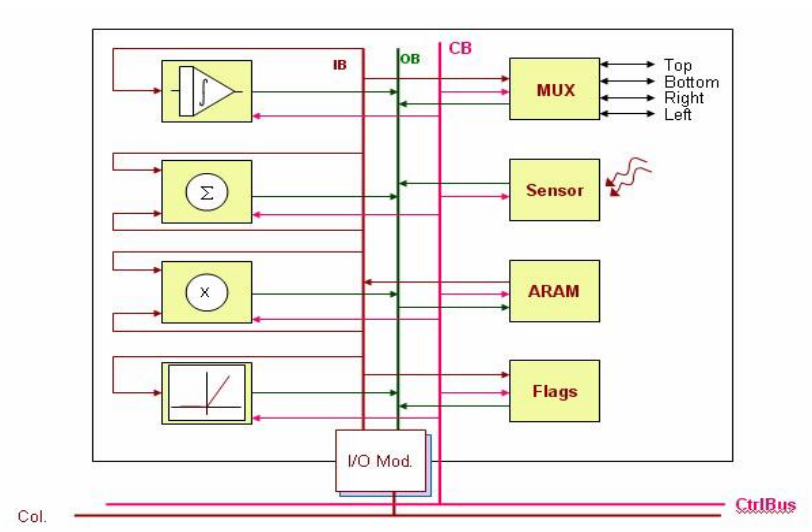

(a)

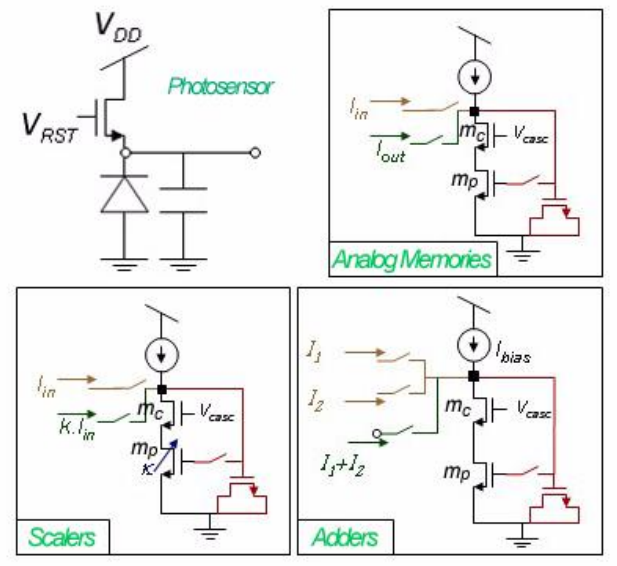

(b)

Figure 2: (a) Cell structure. (b) Analog implementation of some operators in current mode. 
(a)

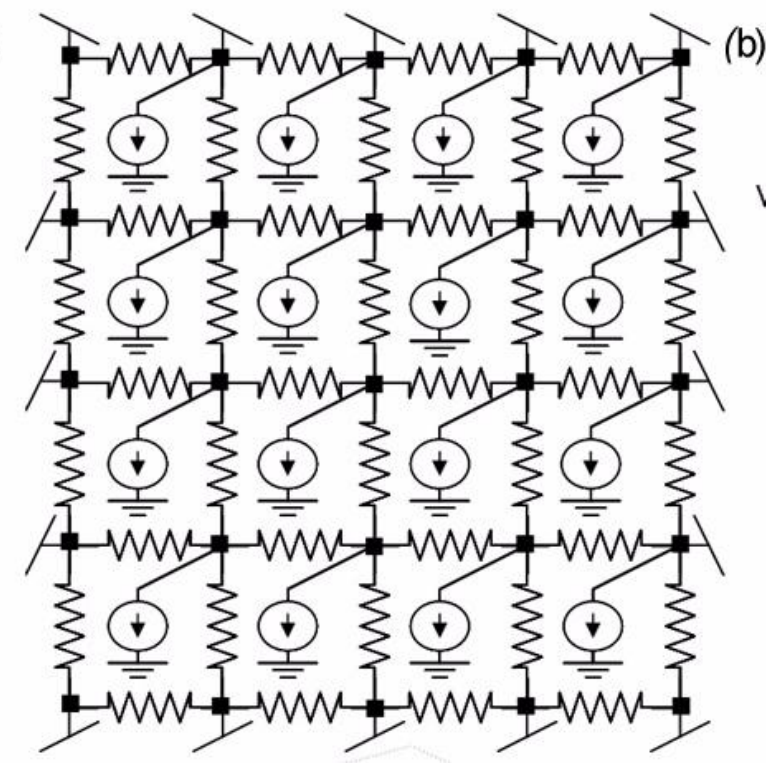

(b)

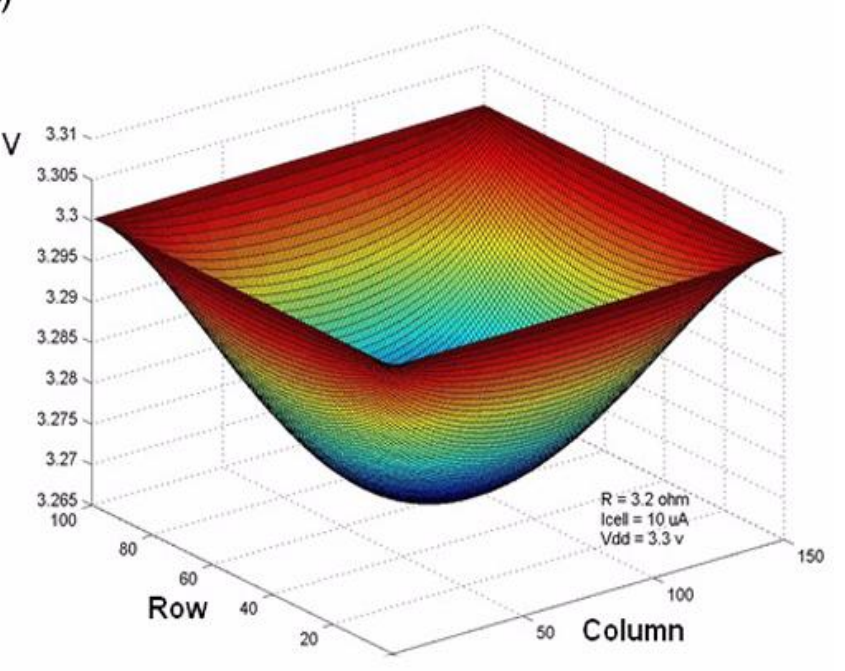

Figure 3: (a) Resistive grid structure used to model the voltage supply curvature. (b) Voltage variation across the array (for the case of an array with the following characteristics: 100x150 photosensors, 3.3 Volts of surrounding voltage supplies, resistances between nodes of $3.2 \Omega$, and a power consumption of $10 \mu \mathrm{A}$ per cell).

The saturation of the photosensors consists of the complete discharging of the integrating capacitor before the exposition time ends. This depends on a number of parameters, namely the incident light power $(P)$, the integration time $(T)$, the oxide capacitance Cox, and the quantum efficiency $\eta^{6}$ (Eq. (1)).

$$
\operatorname{Vph}(T)=\operatorname{Vreset}-\frac{\operatorname{Iph}(T)}{C}, \text { where: } \quad \operatorname{Iph}(T)=\eta \cdot \frac{q A P \lambda}{h c}, \text { and } C=C_{o x} \cdot \mathrm{W} \cdot \mathrm{L}
$$

The saturation of the photosensors is taken into account by imposing the following restriction to $\operatorname{Vph}(T)$ :

$$
0 \leq \operatorname{Vph}(T) \leq \text { Vreset }
$$

Another source of non idealities are the defects in the fabrication process. Here mismatch ${ }^{5}$ is the main error source. The random variation of parameters such as Cox and $\eta$ along the chip will define the influence of this aspect on the performance of the implementation. To model that, a random spatial variation is introduced in those parameters, with gaussian distribution. Notice that the stochastic process does not depend on time, but only in the coordinates of the pixel, being therefore a spatially varying time independent noise, defined by Eq. (3).

$$
\eta_{i, j}=N\left(\eta_{\text {nom }}, \sigma_{\eta}\right) \text { and } C_{o x(i, j)}=N\left(C_{o x(n o m)}, \sigma_{C}\right)
$$

Another important undesired effect that can seriously affect the behaviour of the model is the leakages in analog memories $\left(\Delta V_{l}\right)$. In an analog implementation, a memory may simply consist of a capacitor and a switch. Different parasitic currents (mainly due to the access switch) contribute to discharge the capacitor, thus degrading the stored information. The main parameters that influence this effect are the size of the analog memories $(\mathrm{Wm})$ and the operating temperature (Temp). Voltage drop in such memories is modelled as Eq. (4). Besides, the so called feedthrough effect should also be remarked $\left(\Delta V_{f}\right)^{6}$. This effect consists of a charge injection in the capacitor when a switch (implemented with a MOS transistor) connected to it is turned from ON to OFF. The charge in the transistor's channel is delivered to the nodes at which it is connected, when this channel is destroyed. This generates an injection of negative charge (in the NMOS 
case) in the capacitors attached to the switch, and degrades the data stored in the analog memories, according to Eq. (4). If the operations are carried out in current mode, the translation to current increments should be made, as can be seen in Eq. (5), where $g_{m}$ is the transconductance of the transistors $m_{c}$ and $m_{p}$ in Fig. 2b).

$$
\begin{gathered}
\Delta V_{l} \cong-\left(\frac{I_{\text {leak }}(\text { Temp })}{C_{\text {ox }} \cdot W_{\text {mem }}^{2}}\right) \cdot T_{\text {frame }} \quad \Delta V_{f} \cong-\left(\frac{C_{\text {ox }} \cdot W_{\text {switch }} \cdot L_{\text {switch }} \cdot V_{D D}}{C_{o x} \cdot W_{\text {mem }}^{2}}\right) \\
\Delta I_{t} \cong g_{m} \cdot\left(\Delta V_{l}+\Delta V_{f}\right)
\end{gathered}
$$

Apart from the previous error sources, the influence of temporal noise must be also taken into account. This appears in every electronic circuit, and it results from the addition of several different contributions, as shot noise, flicker noise, thermal noise, etc. ${ }^{6}$ This can be modelled by summing up at each step of the processing an additive gaussian noise. Let $X$ be the dynamic range of a given layer of the model. Then, the expression of a gaussian noise with medium value 0 and an $100 \cdot d \%$ standard deviation would be as shown in Eq. (6).

$$
n=d \cdot X \cdot N(0,1)
$$

\section{3-CASE STUDY}

As an example of this methodology the electronic implementation of the LGMD Model proposed by Rind et al. ${ }^{1}$ is studied. The model represents a neural network structure, decomposed into 4 retinotopically connected layers of neurons that interact with each other, as can be observed in Fig. 4. According to the proposed methodology in Fig. 1, the first step is the computational modelling of the system, which is explained in section 3.1 .

\subsection{Computational description of the model}

The model takes as input the luminance of the current scene (i.e. images are captured in the first layer), and calculates the movement map of the scene, i.e. the difference between the current frame and the previous one, so that a signal proportional to the moving objects is generated. Particularly, its value is high if there has been a luminance change from dark to bright and small if the change has been in the opposite sense. This signal excites the so called ' $E$ ' cells that implement the raw excitation due to activity in the sensing area. The movement map signal is also passed to the inhibitory ' $I$ ' layer, where inhibition to the raw excitation is generated, in order to eliminate the spurious activity and the one due to the background movement. Between the ' $E$ ' and ' $I$ ' layers a critical race between excitation and spread inhibition is accomplished. Thus, if the excitation is strong enough it will go on to the next layer. Otherwise it will be cancelled by inhibition, thus not causing any response in the model. The result of this competitive race between ' $E$ ' and ' $I$ ' cells leads to the signal that feeds the ' $S$ ' cells. This layer is directly presynaptic to the LGMD, and all its neuron's axons meet in the LGMD neuron, where their response is integrated to generate the LGMD potential value. There is also in this neural structure a forward pathway that directly connects the luminance input with the LGMD. The purpose of this Feedforward Inhibition is to eliminate LGMD responses against abrupt excitations, such as sudden changes in the illumination conditions.

The LGMD model has been algorithmically implemented in order to simulate $\mathrm{it}^{2,3}$ and for that purpose, some changes have been made to the original idea. The algorithm, likewise the biological model, takes as input the luminance in the current scenario. The input image has been decided to be of 100x150 pixels size, and its grayscale resolution to be of 6 bits, thus each pixel having values between 0 and 63. This dynamic range has shown to be adequate for this particular application. The input image then, is first passed to the so called ON-OFF layer that calculates the movement map of the current scene. Let $L(i, j, T)$ be the luminance signal captured by the photosensors, where $i$ and $j$ are the row and column index, and $T$ the discrete timesteps. Then, the ON-OFF signal (the movement map), is defined as:

$$
C(i, j, T)=\left|\frac{L(i, j, T)-L(i, j, T-1)}{2}\right|
$$




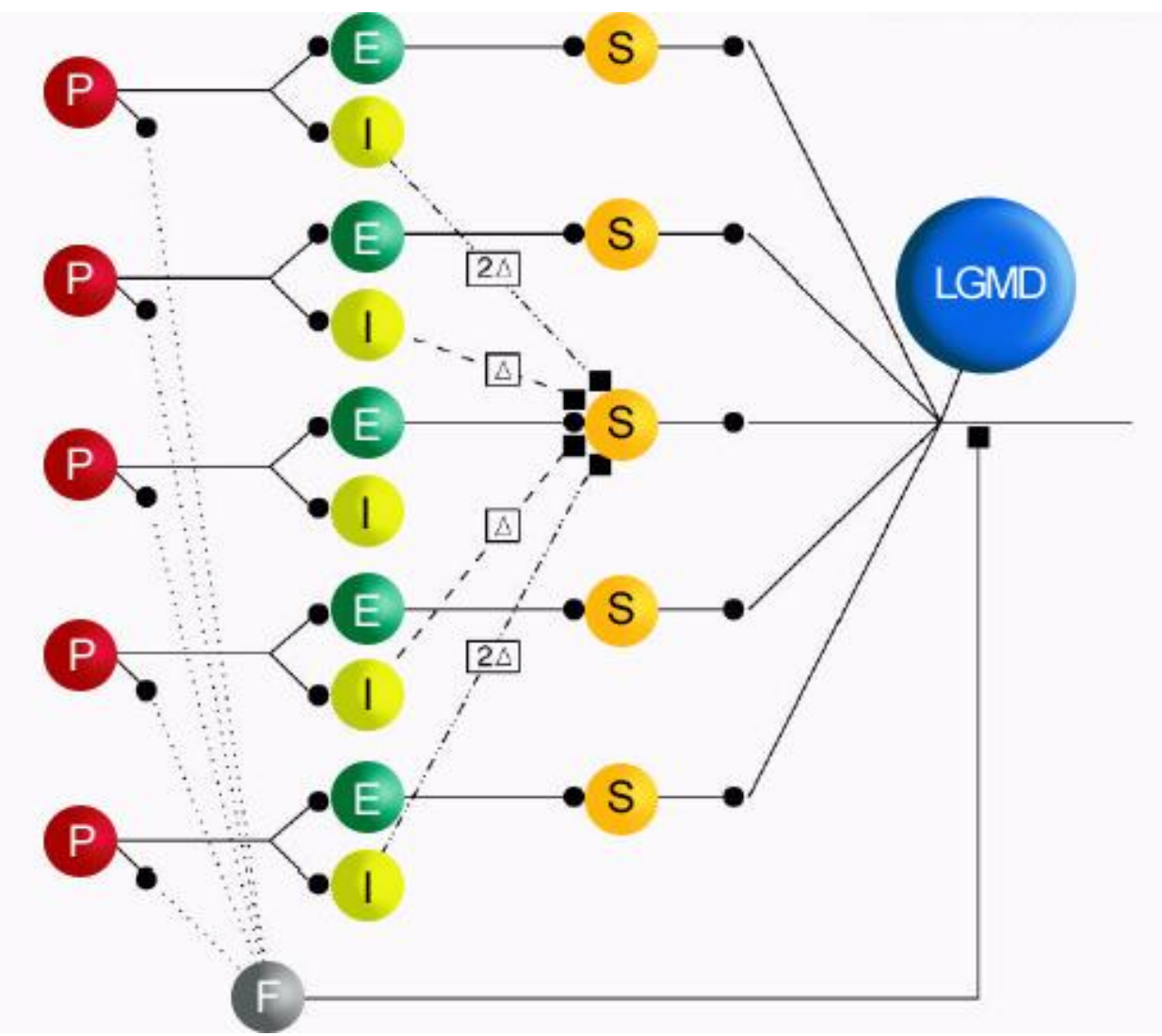

Figure 4: Original Rind et al. Model for the locust's Lobula Giant Movement Detector

where $0<i<100$ is the row index, $0<j<150$ is the column index. Movement map values $C(i, j, T)$ are rounded (so that $C \in[0,31])$ before fed into the subsequent stage, the inhibition layer, which in this case consists simply in an analog memory, as can be seen in Eq. (8).

$$
I(i, j, T)=C(i, j, T)
$$

$I(i, j, T)$ is fed into the next stage, the Summing Cells layer, where the computation of the critical race between excitation and inhibition is accomplished (Eq. (9)). An adaptation mechanism has also been introduced, to adjust the sensitivity of the model to the current level of excitation. It is called Adaptive Inhibition. It consists in the variation of the inhibition influence depending of the number of consecutive spikes that have been generated until the current frame. The corresponding coefficient for the inhibition is represented by $\operatorname{InhCoeff}(k)$ in Eq. (9). The higher the number of consecutive spikes, the stronger the inhibition (i.e., the higher the inhibition coefficient). This is useful to avoid false spikes and therefore, mistakes in the firing of the collision alarm.

$S(i, j, T)=C(i, j, T)-\operatorname{Inh} \operatorname{Coeff}(k) \times I(i, j, T-1)$, with $k$ varying according to the number of consecutive spikes.

From the S layer, the net scalar excitation is calculated, which consists of the sum of all the values of its cells, adequately scaled to get the signal into the proper dynamic range:

$$
e(T)=\frac{\sum_{i, j} S(i, j, T)}{150}
$$


and finally, the LGMD potential $l(T)$ value results from the temporal averaging of $e(T)$, in the following way:

$$
l(T)=L G M D \operatorname{Coeff}(3) \cdot l(T-2)+L G M D \operatorname{Coeff}(2) \cdot l(T-1)+L G M D \operatorname{Coeff}(1) \cdot e(T)
$$

$L G M D C o e f f$ is the coefficients vector for the LGMD potential calculation ${ }^{4}$. The LGMD potential value is compared at each timestep with the value of a dynamic threshold $V(T)$. Whenever the LGMD is above the threshold, one spike is generated and the values $I(T), I(T-1)$ and $I(T-2)$ are set to 0 . Therefore, a new LGMD variable $v(T)$ is needed, not subjected to that reset:

$$
v(T)=L G M D \operatorname{Coeff}(3) \cdot v(T-2)+L G M D \operatorname{Coeff}(2) \cdot v(T-1)+L G M D \operatorname{Coeff}(1) \cdot e(T)
$$

The threshold $V(T)$ is calculated in the following manner:

$$
V(T)=\text { ThreshCoeff } \cdot(v(T-5)+v(T-10)+v(T-14))+a
$$

ThreshCoeff is the coefficients vector for the threshold calculation, and $a$ is the threshold minimum value, that helps to absorb spurious excitation. The collision alarm firing process begins with the spike generation. One spike is fired at every frame in which the LGMD value is greater that the threshold value, as it actually happens in the locusts. If this happens, as mentioned before, the LGMD values of $T, T-1$ and $T-2$ are set to 0 , and the processing continues in the same way with the next frames. If four or more spikes are detected in an interval of 5 frames, then the collision alarm is fired. Otherwise the model remains silent.

\subsection{Analog Implementation of the Macromodel}

From the computational model previously described, the next step in setting up the macromodel for simulation is to identify the operators and their implementation (Fig. 1). According to section 3.1, the operators needed are adders and scalers, and analog memories to store intermediate results and information from previous frames. In this case, operators will be implemented in current mode since currents can be easily added and scaled, and analog memories can also be designed. Furthermore, part of the circuitry used for implementing the different operators can be shared among them since each operation is multiplexed in time, allowing a reduction in the area needed for each cell element. The proposed structure can be observed in Fig. 2b).

Going ahead in the flux diagram of Fig. 1, the typical hardware level effects described in section 2 are incorporated on each processing layer, and the way they affect the behaviour of the analog electronic implementation is analysed. A software tool and a GUI (Graphic User Interface) has been developed in Matlab ${ }^{\mathrm{Tm}}$. This program receives as inputs a number of parameters (see below), and the video to be processed, and returns the ideal LGMD potential evolution, superimposed to the LGMD potential that would be obtained taking into account non-idealities and spurious effects.

The list of parameters the simulator includes, their description and their default value can be found in Table 1 . To calculate the voltage supply distribution over the array in the case of the locust vision chip, the resistances between each node have been estimated supposing a separation between photosensors of approximately $40 \mu \mathrm{m}$, which is the expected pixel pitch. Thus, supposing a square resistance in the technology of $8 \Omega$ /square, the resulting resistance value is $3.2 \Omega$. After simulating such a resistive grid, with $\mathrm{Vdd}(3.3 \mathrm{~V})$ ideal supplies in the peripheral nodes, and a current source on each node that simulates its power consumption (which in this case has been given a value of $10 \mu \mathrm{A}$ ), the obtained spatial distribution of Vdd can be observed in Fig. 3b). The rest of non idealities included are those presented in section 2.

Figure 5 presents the GUI of the simulator, as well as explanations about the information present on it.

The set of videos available for the experiments have been recorded both in real and simulated automotive situations. In total, there are 49 video sequences representing several scenarios, comprising driving on a highway, collisions with a balloon car, pedestrians crossing in front of the car, roundabouts, and so forth. The sequences are 8 bit@100x150 AVI files at 25 fps. In Fig. 6 examples of these videos can be observed. 


\begin{tabular}{|c|c|c|}
\hline Name (units) & Description & Default Value \\
\hline Tframe (seg) & Time between frames at which the video is processed & $40 \mathrm{msec}$ \\
\hline Light Power $\left(\mathrm{W} / \mathrm{m}^{2}\right)$ & Maximum power of the incident light in the scene & $1 \mathrm{~W} / \mathrm{m}^{2}$ \\
\hline Mismatch $(\%)$ & $\begin{array}{c}\text { Expected mismatching level in the hardware } \\
\text { implementation }\end{array}$ & $0 \%$ \\
\hline Noise (\%) & Expected temporally varying noise level & $0 \%$ \\
\hline $\operatorname{Cox}\left(\mathrm{F} / \mu \mathrm{m}^{2}\right)$ & Oxide capacitance in the selected technology & $4.5 \mathrm{fF} / \mu \mathrm{m}^{2}$ \\
\hline$\eta$ & Quantum efficiency of the photosensors & 0.5 \\
\hline Temp $\left({ }^{\circ} \mathrm{C}\right)$ & Operating temperature & $25^{\circ} \mathrm{C}$ \\
\hline Wm (microns) & $\begin{array}{l}\text { Width of the capacitor employed in the analog memories } \\
\text { (square-shaped) }\end{array}$ & $8 \mu \mathrm{m}$ \\
\hline $\mathrm{W}$ (microns) & Width of the switches & $0.4 \mu \mathrm{m}$ \\
\hline L (microns) & Length of the switches & $0.35 \mu \mathrm{m}$ \\
\hline Vdd File & $\begin{array}{l}\text { File with the spatial distribution of the voltage supply, } \\
\text { including loses due to the finite resistance of the wires }\end{array}$ & --- \\
\hline
\end{tabular}

Table 1: Programmable Parameters in the Simulator

\subsection{Experimental results}

According to the flux diagram depicted in Fig. 1, the next step is the simulation of the macromodel. Different experiments can be set up to characterize the influence of non idealities on its behaviour. For instance, execution on test videos sweeping iteratively the range of temperatures in which the model is desired to work properly, or changing the mismatch and temporal noise levels. This is done in order to obtain an empiric quantitative description of the influence of those parameters, to accomplish modifications to the model and/or to the implementation make the system more robust. Besides, the set of specifications on mismatch, temperature, temporal noise, etc. that the implementation must meet can be established. The inclusion of the non idealities is done whether in the whole model, or layer by layer, the latter helping to describe the sensitivity of each layer against the respectively varying parameter. This is also very useful to identify the critical layers that present harsher restrictions for their implementation.

As an example, the experiments to characterize the effect of temporal noise, mismatch, and temperature in the LGMD model are presented. In order to describe the dependence of the LGMD value with respect to the noise and mismatch levels, simulations varying only these two parameters are made. The mean value of the variation in the output of the model along all the frames is calculated. The results are shown in Fig. 7. The mean deviation from the ideal response remains under 10\% with a $20 \%$ of mismatch level. This is a good response of the model against mismatch, but the most important point with respect to that is the way it affects the actual generation of collision alarms. When repeating the simulations and comparing the number of alarms with the ones without noise, the result is that of Fig. 8. The good performance of the model can be better observed there, since the mean number of extra false alarms fired remains below 1 with a mismatch level of $20 \%$. Therefore, setting up a maximum acceptable number of spurious alarms an upper limit for noise and mismatch can be specified from Fig. 8 and Fig. 7.

Temperature is also a critical factor to be considered. The high range of temperature under which this chip should perform correctly makes this parameter specially important. Simulations are run to characterize the model's behaviour with respect to this parameter, varying the temperature in the range $[-4,110]^{\circ} \mathrm{C}$, in steps of $6^{\circ} \mathrm{C}$. This huge range of temperature is a standard specification in the automotive field. The simulations show that the model is not robust against temperature 


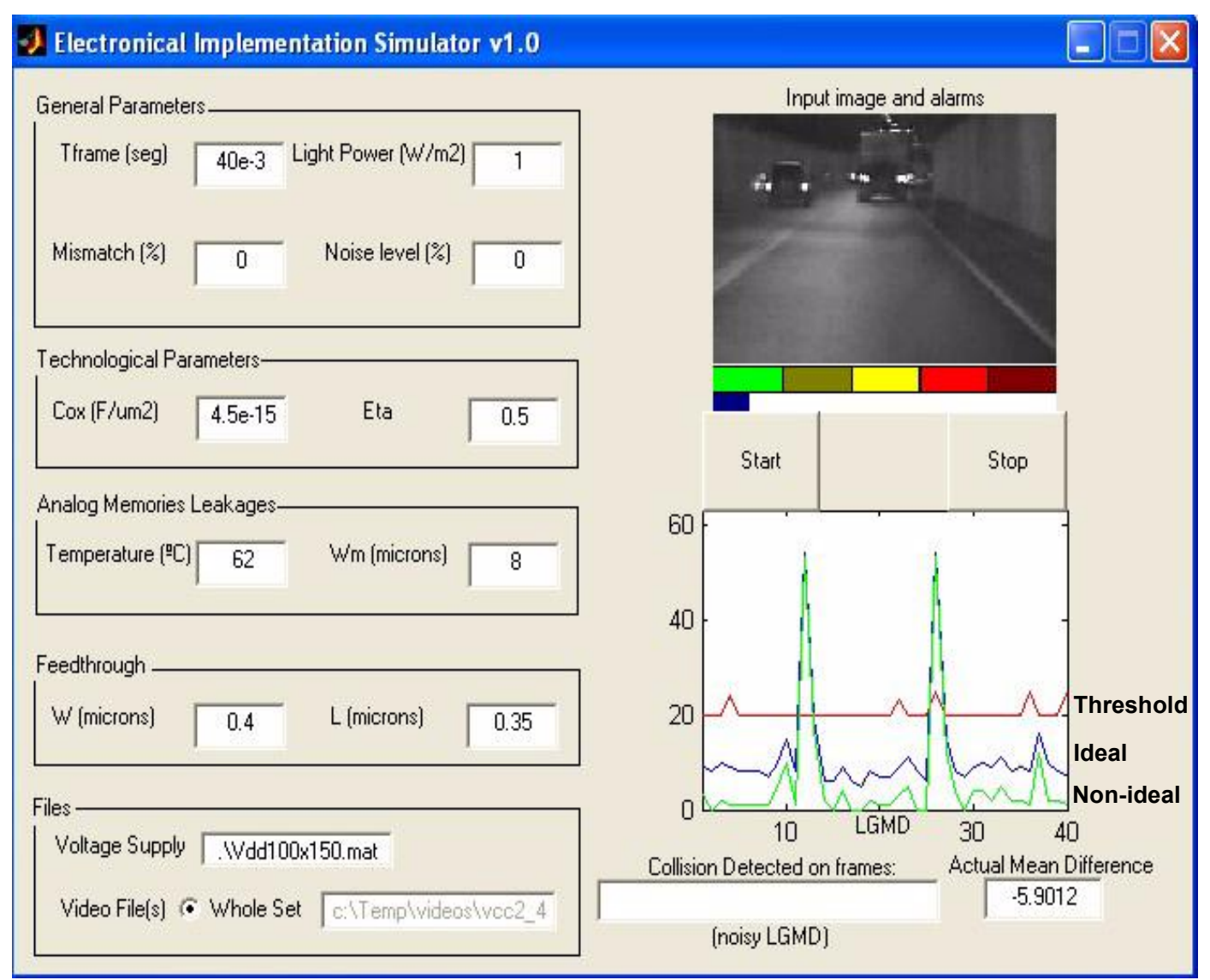

Figure 5: Graphic User Interface of the simulator. On the left side, the values of the configurable parameters are inserted. Output images are displayed on the right side of the window. The upper one is the input video, with a bar representing the current collision alarm status below, from left (no danger), to right (collision alarm). The window below shows the LGMD potential values, the ideal one (in blue), the one including undesired effects due to the electronic implementation (in green), and finally the LGMD potential threshold calculated for the latter (in red). In the two boxes below the frame number at which an alarm is fired and the current cumulative mean difference between the ideal LGMD potential and the one including non-idealities are displayed.

variations, because it does not perform well within the highest part of the temperature range, as can be seen in Fig. 9, due to the huge leakage on analog memories at very high temperatures. In this case, given that the specifications are not met, the designer should go back into the design loop depicted in Fig. 1 trying to overcome this drawback. For instance, resizing the analog memories would reduce the effect on current leakages. If redesign does not give positive results then the computational description of the model must be modified, to make it more robust. One of the possibilities would be to store only one timestep of memory instead of two, and include a new layer of processing, thus entering the redesign loop in the computational description step instead of in the design step in Fig. 1. This iterative process leads to an optimum set of analog electronic specifications that would serve as starting point for circuit designers in the final integration of the system in silicon. 

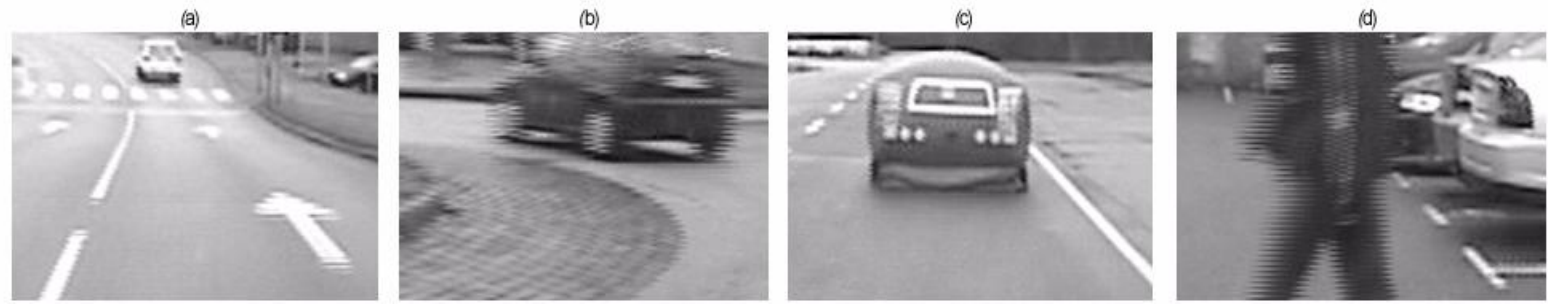

Figure 6: Examples of test videos: (a) Driving on a highway. (b) Roundabout. (c) Collision with test balloon car. (d) Pedestrian crossing in front of the car.

\section{4-CONCLUSIONS}

Using the previously presented methodology, restrictions and specifications on the electronic characteristics of the model to be integrated can be set, serving as starting point for the circuit design stage. The maximum acceptable values for the proper functioning of the model of undesired hardware effects as mismatching, feedthrough and temporal noise can be obtained from the experiments. Behavioural simulations including those effects can be easily accomplished to predict that the chip will respond in an adequate way. It can also be analysed which parts of the model are more sensitive to random variations in the parameters, or different working conditions, and with that, retune them to be more robust. This methodology allows to find specifications for analog circuitry in a reliable and agile way.

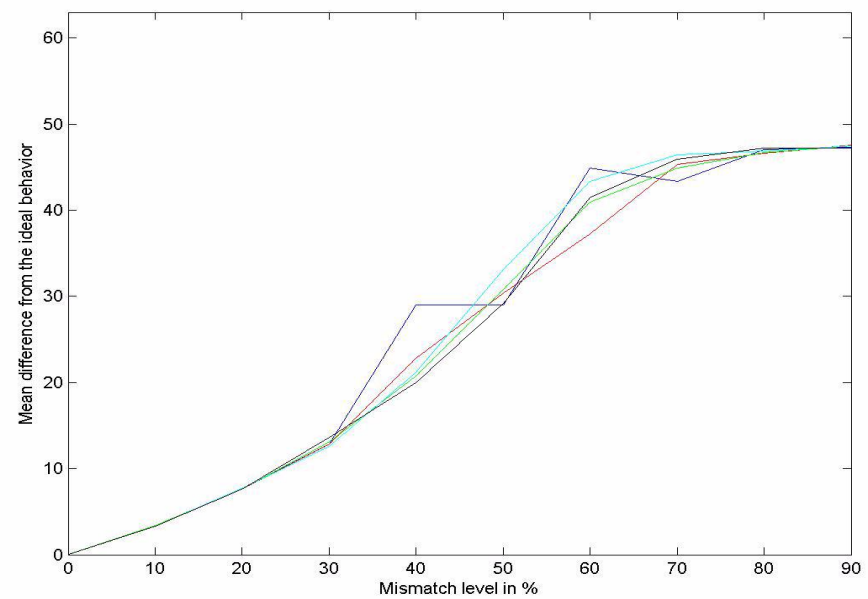

Figure 7: Dependence of the LGMD potential value with noise and mismatch level. In the $\mathrm{x}$ axis there is the mismatch level in \%. The temporally varying random noise level is 10 times smaller than the mismatch level. In the y axis the mean difference between the LGMD value with and without noise and mismatch. 


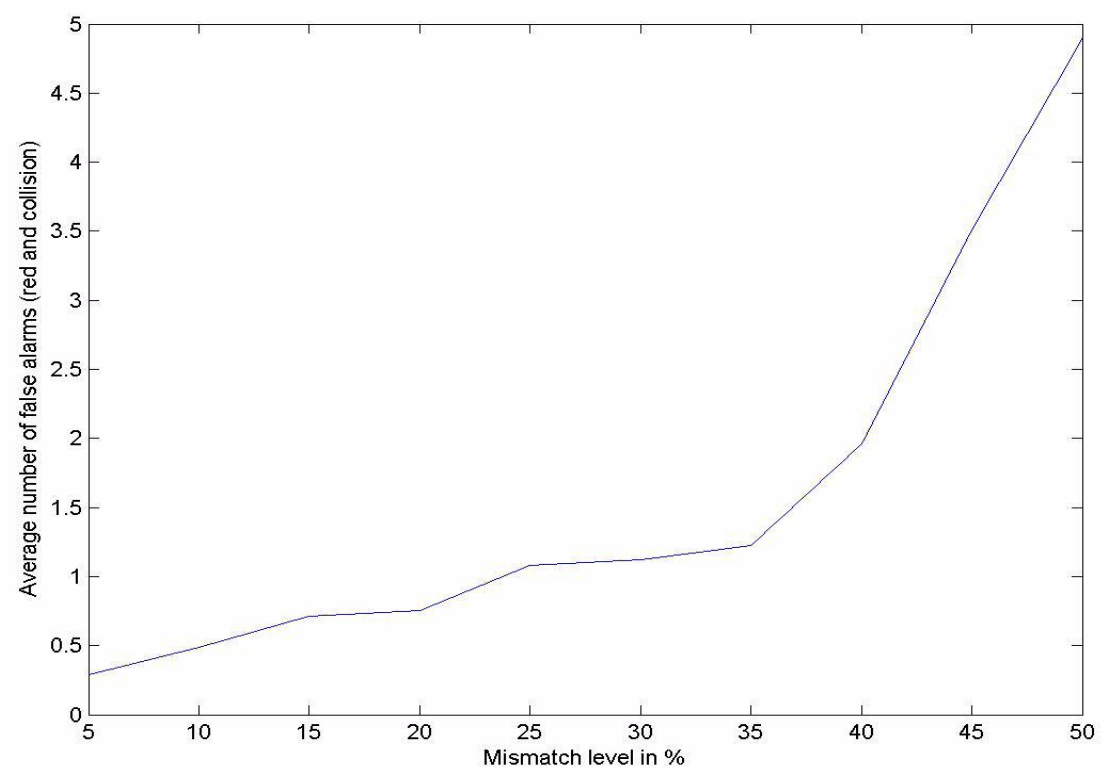

Figure 8: False collision alarms due to the influence of mismatch and noise. See Figure 7 for axis description.

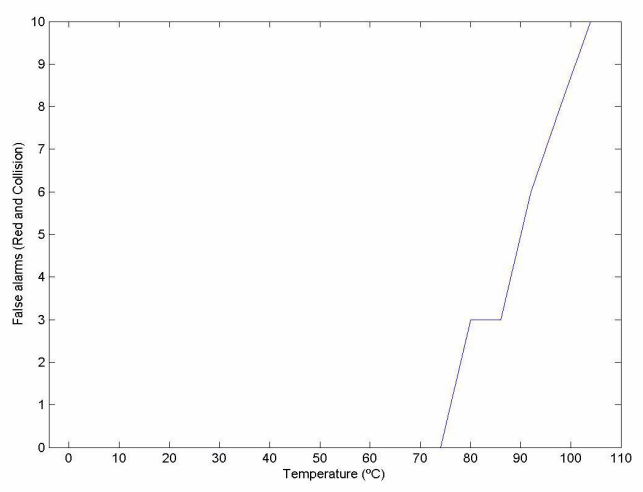

(a)

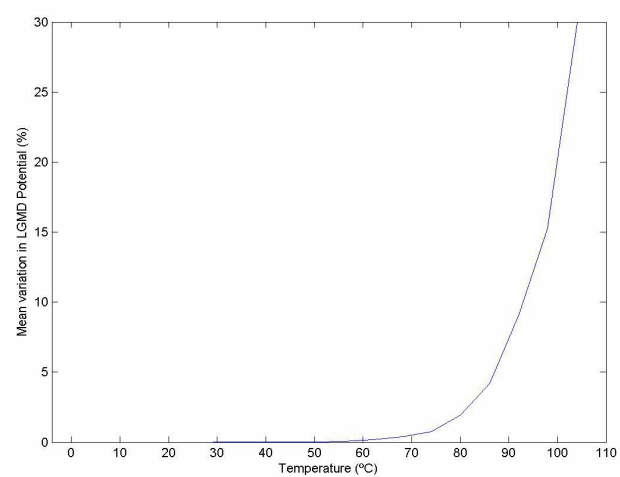

(b)

Figure 9: (a) Number of spurious collision alarms with respect to the working temperature. (b) Influence of temperature on the LGMD potential. Both graphs are the medium value for all the videos and all the frames.

\section{ACKNOWLEDGEMENTS}

This paper is partially funded by the European Project IST-2001-38097 (LOCUST), and TIC2003 - 09817-C02-01 (VISTA). Mr. Cuadri's work is funded by an F.P.U. grant from the Spanish Ministry of Education and Science. The authors would like to thank Dr. F.C. Rind, Dr. Richard Stafford and their group in the University of Newcastle-upon-Tyne for their work in the modelling of the locust's visual system. Special thanks also to Volvo Car Corporation for recording the test videos representing automotive situations. 


\section{REFERENCES}

1.F.C. Rind and P.J. Simmons, "Seeing what is coming: building collision-sensitive neurones". Trends Neuroscience 22(5), pp 215-220. May 1999.

2.Project IST-2001-38097 (LOCUST), Deliverable 11, "Biological Model Report".

3.J.Cuadri, G.Liñán, R.Stafford, M.S.Keil, E.Roca, "A bioinspired collision detection algorithm for VLSI implementation", SPIE Europe International Symposium, Microtechnologies for the New Millennium, to be held on 9 - 11 May 2005, Sevilla, Spain.

4.Yue S., Rind F.C., Keil M.S., Cuadri J. \& Stafford R. "A bio-inspired visual collision detection mechanism for cars: Optimisation of a model of a locust neuron to a novel environment", submitted to Neurocomputing, October 2004.

5.Pelgrom, M.J.M.; Duinmaijer, A.C.J.; Welbers, A.P.G., "Matching properties of MOS transistors"; IEEE Journal of Solid-State Circuits, Volume: 24 , Issue: 5 , Oct. 1989, Pages:1433 - 1439.

6.Orly Yadid-Pecht, Ralph Etienne-Cummings, “CMOS Imagers: From Phototransduction to Image Processing “, Kluwer Academic Publishers, 2004. ISBN: 1-4020-7961-3. 